\title{
Fatal case of extensively drug-resistant Mycobacterium tuberculosis Beijing genotype infection in an injecting drug user, Athens, Greece, 2012
}

K Leuow ${ }^{1,2}$, D Papaventsis (dpapaventsis@gmail.com) ${ }^{2,3}$, S Kourkoundi ${ }^{1}$, P loannidis ${ }^{3}$, S Karabela ${ }^{3}$, S Tsikrika ${ }^{4}$, I Marinou ${ }^{3}$,

A Papavasileiou ${ }^{4}$, M Stone 5,6 , F Drobniewski ${ }^{5,6}$, V Paparisos ${ }^{1}$, E Vogiatzakis ${ }^{3}$

1. HIV-Unit, 'Andreas Sygros' University Hospital for Dermatology and Venerology, Athens, Greece

2. These authors contributed equally to this work

3. Microbiology Department, National Reference Laboratory for Mycobacteria, Athens, Greece

4. Clinic for Multidrug-Resistant Tuberculosis, 'Sotiria' Chest Diseases Hospital, Athens, Greece

5. National Mycobacterium Reference Laboratory, Health Protection Agency, London, United Kingdom

6. Barts and the London Queen Mary's School of Medicine and Dentistry, London, United Kingdom

Citation style for this article:

Leuow K, Papaventsis D, Kourkoundi S, loannidis P, Karabela S, Tsikrika S, Marinou I, Papavasileiou A, Stone M, Drobniewski F, Paparisos V, Vogiatzakis E. Fatal case of extensively drug-resistant Mycobacterium tuberculosis Beijing genotype infection in an injecting drug user, Athens, Greece, 2012. Euro Surveill.

2013;18(12):pii=20430. Available online: http://www.eurosurveillance.org/ViewArticle. aspx?Articleld=20430

We present the first fatal case of extensively drugresistant tuberculosis (XDR-TB) in an injecting drug user (IDU) in Athens, Greece, co-infected with human immunodeficiency virus and hepatitis $C$ virus and discuss the implications for public health. Despite immediate initiation of treatment, the patient's condition gradually deteriorated and he died 16 days after hospital admission because of multiple organ failure. The contact tracing investigation revealed no further infections among the patient's contacts.

We report the first fatal case of extensively drugresistant tuberculosis (XDR-TB) in an injecting drug user (IDU) in Athens, co-infected with human immunodeficiency virus (HIV) and hepatitis C virus (HCV) and discuss the implications for public health in times of austerity in Greece.

Since 2011, Greece has been facing a considerable outbreak of HIV infections among IDU in Athens. Although concerns about migration have been raised, there has been no evidence that HIV is a migrant-specific issue, except for migrants who inject drugs [1].

XDR-TB is defined as resistance to rifampicin and isoniazid (multidrug-resistant tuberculosis; MDR-TB) plus resistance to a fluoroquinolone and at least one of the three injectable second-line drugs (SLD): amikacin, kanamycin, capreomycin [2]. XDR-TB constitutes an emerging issue in Europe and a significant public health problem in countries of the former Soviet Union (FSU) $[3,4]$. It has been identified in 84 countries and the average proportion of MDR-TB cases with XDR-TB worldwide was 9.0\% (range 6.7-11.2\%) in 2012 [5]. XDR-TB has been associated with HIV in countries such as Latvia and Ukraine [4]. Treatment outcomes have been significantly worse for patients with drug-resistant TB, as therapeutic options are limited, less effective, more toxic and costly [5].

In Greece, TB incidence is underreported [6]. From 2006 to 2009, 13 XDR-TB cases were recorded at the National Reference Laboratory for Mycobacteria (NRLM) in Athens: nine cases among Greek nationals, most of them repatriated from the FSU and the remaining four cases were recorded among migrants. XDR-TB ratio among MDR-TB cases was 19\% [7].

\section{Case report}

On 18 October 2012, a man in his early 40 s originating from Ukraine, was admitted to the HIV Unit, 'AndreasSygros' University Hospital, in Athens, Greece. The patient presented with fever, weight loss, abdominal pain, diarrhoea and fatigue. He had lived for more than a decade in Greece and had a medical history of intravenous drug abuse, HIV (Centers for Disease Prevention and Control (CDC) HIV classification subcategory $\mathrm{B}_{3}$ ) and HCV co-infection. Due to poor compliance to antiretroviral therapy, his HIV infection remained uncontrolled and he developed resistance to multiple antiretroviral drugs. In February 2012, he had a CD4 count of 25 cells/uL and a viral load of 39,000 copies/ $\mathrm{mL}$. One month before his admission to hospital, the patient had returned to Greece from a six-month visit to Ukraine.

Upon admission, physical examination revealed hepatosplenomegaly and diffuse abdominal tenderness. Auscultation of thorax revealed bilateral crackles. Blood pressure was $110 / 60 \mathrm{mmHg}$, SatO ${ }_{2} 97 \%, 82 \mathrm{bpm}$, and breaths 26 per minute. Computed tomography (CT) revealed interlobular and bronchial thickening of lower lung lobes, with a calcified granuloma of the left lower lobe and lymph nodes of the left pulmonary hilum and 
in the sub-cardinal area, a small pleural effusion (left) and enlarged lymph nodes in the Haller's tripod and the hepatic hilum. Laboratory investigation showed mild anaemia, increased erythrocyte sedimentation rate (ESR), gamma-glutamyl transferases (gamma-GT), serum alkaline phosphatase (SAP), lactate dehydrogenase (LDH) and hypoalbuminemia.

Highly active antiretroviral therapy (HAART) was reinitiated immediately consisting of raltegravir, atazanavir, ritonavir and $A Z T / 3 T C / A B C$ (with intravenous trimethoprim-sulfamethoxazole (TMP-SMX) as prophylactic treatment for Pneumocystis carinii). Blood and gastric fluid specimens were sent to NRLM for acid fast bacilli (AFB) microscopy and mycobacterial culture. Smears did not reveal AFB. However, due to the advanced HIV condition, the clinical presentation, the geographic origin and recent travel history, and the severity of the patient's condition, empiric therapy was initiated against both Mycobacterium tuberculosis and M. avium. Tuberculin skin test (TST) and Quantiferon TB Gold IT testing (Cellestis, Australia) were negative, but these results were attributed to the patient's immunosuppression. The patient was administered rifabutin, isoniazid, ethambutol orally, and intravenous clarithromycin and amikacin.

Despite treatment, the patient's condition gradually deteriorated. Due to severe anaemia and leucopoenia, a bone marrow examination was performed to rule out a lymphoproliferative disorder, and histology indicated a mycobacterial infection. Bone marrow smears did not show AFB. An abdominal CT scan showed hepatomegaly, lesions in the spleen, and a considerable amount of free peritoneal fluid in the pelvis minor. Fifteen days after initiation of treatment, mycobacterial growth was detected in gastric fluid and blood by automated liquid culture (BD Bactec MGIT 960, Maryland, USA). After one more day the patient died of multiple organ failure.

\section{Molecular analyses}

M. tuberculosis complex was identified using Genotype MTBDRplus v.2 (Hain Lifescience, Nehren, Germany). The assay revealed mutations $\mathrm{S}_{531} \mathrm{~L}$ (rpoB gene) and $\mathrm{S}_{315} \mathrm{~T}_{1}$ (katG gene), confirming resistance to rifampicin and isoniazid, respectively. Mutations $\mathrm{D} 94 \mathrm{G}$ (gyrA gene) and A1401G (rrs gene), conferring resistance to fluoroquinolones and injectable SLD, respectively, were found by the Genotype MTBDRsl assay (Hain Lifescience, Germany). This profile was consistent with XDR-TB, and the HIV Unit was informed postmortem. MIRU-VNTR 24-loci genotyping was requested from the World Health Organization / Global Laboratory Initiative (WHO/GLI) Supranational Mycobacterium Reference Laboratory, Health Protection Agency, London, United Kingdom. The isolate belonged to the single 'East European' subtype of the Beijing lineage that is present in Estonia and widely in eastern Europe [8]. Phenotypic drug susceptibility testing (DST) using the modified proportion method on solid and liquid media confirmed molecular DST results (Table).
TABLE

Drug susceptibility testing results, fatal case of extensively drug-resistant tuberculosis in an injecting drug user, Athens, Greece, 2012

\begin{tabular}{|l|c|}
\hline Drug & Resistant (R) / Sensitive (S) \\
\hline Isoniazid o.2 ug/mL & $R^{a}$ \\
\hline Rifampicin $40 u g / m L$ & $R^{a}$ \\
\hline Ethambutol $2 u g / m L$ & $R^{a}$ \\
\hline Pyrazinamide $100 u g / m L$ & $R^{b}$ \\
\hline Streptomycin $5 u g / m L$ & $R^{a}$ \\
\hline Amikacin $1 u g / m L$ & $R^{a}$ \\
\hline Ofloxacin $2 u g / m L$ & $R^{a}$ \\
\hline Capreomycin $40 u g / m L$ & $R^{a}$ \\
\hline Cycloserine $40 u g / m L$ & $R^{a}$ \\
\hline Ethionamide $40 u g / m L$ & $R^{a}$ \\
\hline
\end{tabular}

a Indirect proportion method on solid media (Löwenstein-Jensen). ${ }^{\mathrm{b}}$ MGIT 960.

\section{Contact tracing investigation}

Investigation of the patient's contacts included four close family contacts. Family contacts and healthcare workers in the HIV unit of the hospital underwent a comprehensive individual risk assessment with chest X-ray, TST or Quantiferon TB Gold IT testing at the Clinic for Multidrug Resistant Tuberculosis, Athens, following the European guidelines [9]. All contacts were informed about the risks and symptoms, and were provided with easy access to the clinic for regular clinical observation. No evidence of infection was found as of 15 March 2013.

\section{Discussion}

TB control is facing major challenges worldwide. Co-infection with HIV (TB/HIV) and MDR/XDR TB make control activities more complex and demanding. Treatment options for XDR-TB are extremely limited because SLD are less effective, more toxic, and more costly than first-line therapies [10]. In immunocompromised patients, XDR-TB is devastating; in a study from South Africa, 52/53 patients died, with median survival of 16 days from time of diagnosis [11]. TB and HIV among IDU are converging with hepatitis C, further complicating the management of cases [12] .

To our knowledge, this is the first report of a fatal XDR-TB Beijing genotype disseminated infection in an IDU co-infected with HIV and HCV in Greece. HIVrelated TB continues to increase even in countries with well-organised national TB control programmes that are implementing the directly observed treatment short-course (DOTS). The Tuberculosis Committee of the Hellenic Center for Disease Control and Prevention 
(HCDCP) worked on a National Tuberculosis Control Program which was completed in 2007 and since then has been part of the National Action Plan to Prevent Communicable Diseases, 2008-2012. The Committee has made suggestions for improvement in terms of TB underreporting and synchronisation between the services controlling TB, but these have not been fully put into practice [13]. Immigration from areas with high MDR/XDR-TB incidence and the effects of the current economic crisis, in particular increased unemployment and numbers of homeless people, along with budget cuts in prevention services and in services targeted at vulnerable and hard to reach population groups, add to already existing challenges to the control of TB including MDR/XDR-TB as well as to prevention and control of HIV.

The primary strategy for controlling and preventing TB includes rapid disease diagnosis and initiation of treatment. Speed of detection is important for public health. Diagnosis of active TB by microscopy or culture can be more difficult in HIV patients, as their bacterial load is usually lower [14]. Gene Xpert MTB/RIF (Cepheid, Sunnyvale, (A), a rapid molecular assay endorsed by the WHO in December 2010 for the simultaneous detection of TB and rifampicin resistance, is recommended to be used as the initial diagnostic test in individuals suspected of MDR/XDR-TB or HIV/TB co-infection [15]. In Greece and in a number of other European countries, the Xpert MTB/RIF assay has not been introduced in clinical routine yet because of its high costs [16].

Collaborative TB/HIV activities should be applied at European level to reduce TB burden among people living with HIV and the burden of HIV among TB patients [17]. The threats to public health emerging from the spread of MDR/XDR-TB among HIV individuals should make TB case detection, treatment and prevention a priority for the national AIDS control programmes. Apart from active case finding using innovative TB diagnostic assays, other interventions such as early initiation of antiretroviral therapy, strengthening HIV and TB surveillance and implementing joint recording and reporting formats, scaling-up uptake of cotrimoxazole preventive therapy and antiretroviral therapy, and last but not least, implementing WHO/ United Nations Office on Drugs and Crime (UNODC)/ Joint United Nations Programme on HIV/AIDS (UNAIDS) guidelines for addressing TB/HIV in people who inject drugs [18], should save lives in the future.

\section{Acknowledgements}

We would like to thank our colleagues from the HIV Unit, 'Andreas Sygros' University Hospital for Dermatology and Venerology, the Microbiology Department - National Reference Laboratory for Mycobacteria, the Clinic for Multidrug Resistant Tuberculosis, 'Sotiria' Chest Diseases Hospital, Athens, Greece and the National Mycobacterium Reference Laboratory, Health Protection Agency, Barts and the London Queen Mary's School of Medicine and Dentistry, London, United Kingdom for their support.
Conflict of interest

None declared.

Authors' contributions

$\mathrm{KL}$ and DP wrote the manuscript including conception and design, acquisition, analysis and interpretation of data. SK provided clinical care and participated in data collection. PI carried out molecular analysis and made helpful comments. SK supervised mycobacterial culture, DST and Quantiferon testing. ST performed contact tracing investigation. IM carried out liquid culture and DST. AP supervised contact tracing investigation and made useful comments. MS performed MIRU VNTR genotyping. FD, VP and EV revised the manuscript critically and gave final approval of the version to be submitted for publication. All authors read and approved the final manuscript.

\section{References}

1. European Centre for Disease Prevention and Control (ECDC). Joint technical mission: HIV in Greece 28-29 May 2012. Stockholm: ECDC; 2013. Available from: http://ecdc.europa.eu/ en/publications/publications/hiv-joint-technical\%2omissionhiv-in-greece.pdf

2. Manissero D, Fernandez de la Hoz K. Surveillance methods and case definition for extensively drug resistant TB (XDR-TB) and relevance to Europe: summary update. Euro Surveill. 2006;11(44): pii=3070. Available from: http://www. eurosurveillance.org/ViewArticle.aspx?Articleld =3070

3. Devaux I, Manissero D, Fernandez de la Hoz K, Kremer K, van Soolingen D, on behalf of the EuroTB network. Surveillance of extensively drug-resistant tuberculosis in Europe, 2003-2007. Euro Surveill. 2010;15(11):pii=19518. Available from: http:// www.eurosurveillance.org/ViewArticle.aspx?Articleld $=19518$

4. World Health Organization (WHO)/International Union Against TB and Lung Disease Global Project on Anti-Tuberculosis Drug Resistance Surveillance. Anti-Tuberculosis Drug Resistance in the World: Fourth Global Report. Geneva: WHO. 2008. Available from: http://www.who.int/tb/publications/2008/ drs_report4_26febo8.pdf

5. World Health Organization (WHO). Global Tuberculosis Report 2012. Geneva: WHO; 2012. Available from: http://www.who. int/tb/publications/global_report/gtbr12_main.pdf

6. Lytras T, Spala G, Bonovas S, Panagiotopoulos T. Evaluation of Tuberculosis Underreporting in Greece through Comparison with Anti-Tuberculosis Drug Consumption. PLoS ONE. 2012;7(11):e50033. http://dx.doi.org/10.1371/journal. pone.0050033. PMid:23185524 PMCid:3503712.

7. Papaventsis D, Nikolaou S, Karabela S, Ioannidis P, Konstantinidou E, Marinou I, et al. Tuberculosis in Greece: bacteriologically confirmed cases and anti-tuberculosis drug resistance, 1995-2009. Euro Surveill. 2010;15(28):pii=19614. Available from: http://www.eurosurveillance.org/ViewArticle. aspx?Articleld=19614. PMid:20650053.

8. Casali N, Nikolayevskyy V, Balabanova Y, Ignatyeva O, Kontsevaya I, Harris SR, et al. Microevolution of extensively drug-resistant tuberculosis in Russia. Genome Res. 2012; 22(4):735-45. http://dx.doi.org/10.1101/gr.128678.111. PMid:22294518. PMCid:3317155.

9. European Centre for Disease Prevention and Control (ECDC). Management of contacts of MDR TB and XDR TB patients. Stockholm: ECDC; 2012. Available from: http://www.ecdc. europa.eu/en/publications/publications/201203-guidancemdr-tb-contacts.pdf

10. Jacobson KR, Tierney DB, Jeon CY, Mitnick CD, Murray MB. Treatment outcomes among patients with extensively drug-resistant tuberculosis: systematic review and metaanalysis. Clin Infect Dis. 2010;51(1):6-14. http://dx.doi. org/10.1086/653115. PMid:20504231.

11. Gandhi NR, Moll A, Sturm AW, Pawinski R, Govender T, Lalloo $\mathrm{U}$, et al. Extensively drug-resistant tuberculosis as a cause of death in patients coinfected with tuberculosis and HIV in a rural area of South Africa. Lancet 2006; 368(9547):1575-80. http://dx.doi.org/10.1016/S0140-6736(06)69573-1.

12. Taylor Z, Nolan CM, Blumberg HM; American Thoracic Society Centers for Disease Control and Prevention; Infectious Diseases Society of America. Controlling tuberculosis in the United States. Recommendations from the American Thoracic 
Society, CDC, and the Infectious Diseases Society of America.

MMWR Recomm Rep. 2005; 54 (RR-12):1-81. PMid:16267499.

13. Konstantopoulos S. Tuberculosis Control Program. HCDCP

Newsletter. Athens: 16 Nov 2011. Available from: http://www2. keelpno.gr $/ \mathrm{blog} / ? \mathrm{p}=775$ \&lang $=$ en

14. Drobniewski F, Nikolayevskyy V, Balabanova Y, Bang

D, Papaventsis D. Diagnosis of tuberculosis and drug

resistance: what can new tools bring us? Int J Tuberc Lung Dis. 2012;16(7):860-70. http://dx.doi.org/10.5588/ijtld.12.0180. PMid:22687497.

15. World Health Organization (WHO). Policy statement: automated real-time nucleic acid amplification technology rifampicin resistance: Xpert MTB/RIF system Geneva: WHO; 2011. Available from: http://whqlibdoc.who.int/ publications/2011/9789241501545_eng.pdf

16. Papaventsis D, loannidis P, Vogiatzakis ED. Tuberculosis diagnosis update: The new gene Xpert MTB/RIF assay. HCDCP Newsletter. Athens: 16 Nov 2011. Available from: http://www2. keelpno.gr/blog/?p=765\&lang=en

17. World Health Organization (WHO). WHO policy on collaborative TB/HIV activities: Guidelines for national programmes and other stakeholders. Geneva: WHO; 2012. Available from: http://whqlibdoc.who.int/publications/2012/9789241503006 eng.pdf

18. World Health Organization (WHO), United Nations Office on Drugs and Crime (UNODC), Joint United Nations Programme on HIV/AIDS (UNAIDS). Policy Guidelines for collaborative TB and HIV activities for injecting and other drug users, an integrated approach. Geneva: WHO. 2008. Available from: http:// whqlibdoc.who.int/publications/2008/9789241596930_eng. $\mathrm{pdf}$ 